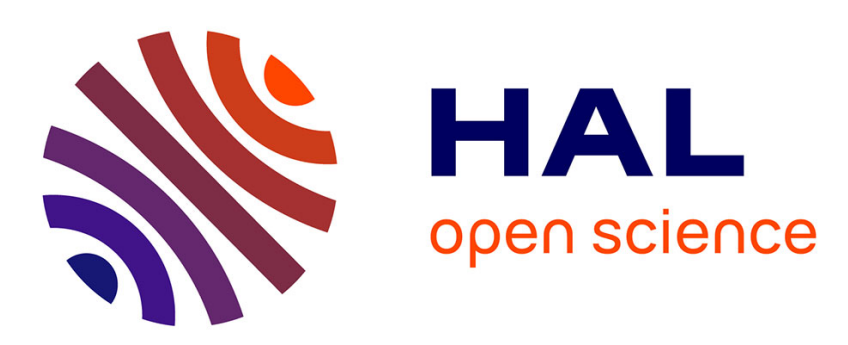

\title{
Oxygen cyclotron harmonic waves in the deep plasmasphere during magnetic storms
}

O A Pokhotelov, D O Pokhotelov, F. Z. Feygin, V. Gladychev, Michel Parrot, K. Hayashi, J. Kangas, K. Mursula

\section{- To cite this version:}

O A Pokhotelov, D O Pokhotelov, F. Z. Feygin, V. Gladychev, Michel Parrot, et al.. Oxygen cyclotron harmonic waves in the deep plasmasphere during magnetic storms. Journal of Geophysical Research Space Physics, 1997, 102, pp.77-83. insu-03235174

\section{HAL Id: insu-03235174 \\ https://hal-insu.archives-ouvertes.fr/insu-03235174}

Submitted on 25 May 2021

HAL is a multi-disciplinary open access archive for the deposit and dissemination of scientific research documents, whether they are published or not. The documents may come from teaching and research institutions in France or abroad, or from public or private research centers.
L'archive ouverte pluridisciplinaire HAL, est destinée au dépôt et à la diffusion de documents scientifiques de niveau recherche, publiés ou non, émanant des établissements d'enseignement et de recherche français ou étrangers, des laboratoires publics ou privés. 


\title{
Oxygen cyclotron harmonic waves in the deep plasmasphere during magnetic storms
}

\author{
O. A. Pokhotelov, ${ }^{1}$ D. O. Pokhotelov, ${ }^{1}$ F. Z. Feygin, ${ }^{1}$ V. A. Gladychev, ${ }^{1}$ \\ M. Parrot, ${ }^{2}$ K. Hayashi, ${ }^{3}$ J. Kangas, ${ }^{4}$ and K. Mursula ${ }^{4}$
}

\begin{abstract}
A new approach to the generation of storm-associated ULF waves with discrete spectra (ion cyclotron harmonic waves) observed in the equatorial plasmasphere is presented. It is proposed that the appearance of waves with phase velocities smaller than the Alfvén velocity is connected with a strong dispersion of magnetosonic waves near the bi-ion frequency occurring in the presence of oxygen ions of ionospheric origin. The waves are generated by an instability involving hot oxygen ions with loss cone or ring like distributions. Such ions are found in the magnetosphere during magnetic storms. A simple analytical model of this instability is elaborated. It is shown that ULF wave observations on board Akebono satellite are in a reasonable agreement with the present theoretical approach.
\end{abstract}

\section{Introduction}

A long time ago Russell et al. [1970] observed ULF (ultralow frequency) emissions on board the OGO 3 satellite in the equatorial plasmasphere. The wave spectrum was localized near the harmonics of proton gyrofrequency. The waves propagated almost perpendicular to the geomagnetic field. The localization of emission, the direction of propagation and polarization were in agreement with the theory of magnetosonic wave propagation in a toroidal waveguide inside the plasmasphere [e.g., Guglielmi et al., 1975; Kaladze et al., 1976; Perraut et al., 1982; Korth et al., 1984; McClements, 1996]. This phenomenon was recently reviewed by Guglielmi and Pokhotelov [1996]. This interpretation was further verified by measurements of these waves on IMP 6, Hawkeye 1 and Explorer 45 satellites [Taylor et al., 1975; Gurnett, 1976; Kintner and Gurnett, 1977]. Harmonically related ULF emissions were

\footnotetext{
'Institute of Physics of the Earth, Moscow, Russia.

${ }^{2}$ Laboratoire de Physique et Chimie de l'Environnement, CNRS, Orléans, France.

${ }^{3}$ Department of Earth and Planetary Physics, University of Tokyo, Tokyo, Japan.

${ }^{4}$ Department of Physical Sciences, University of Oulu, Finland.
}

Copyright 1997 by the American Geophysical Union.

Paper number 96JA03067.

0148-0227/97/96JA-03067\$09.00 also observed by GEOS 1 and 2 [Perraut et al., 1978, 1982; Laakso et al., 1990] and DE 1 satellites [Olsen et al., 1987; Gurnett and Inan, 1988] at higher altitudes ( $L=4-8)$.

The specific feature of the toroidal waveguide surrounding the Earth is that there is no ordinary mechanism of dissipation, for example, absorption of waves at the ends of geomagnetic field tubes. Therefore even a fairly small enhancement above marginal stability of ring current ions may lead to exponential growth of the wave amplitude. The necessary condition of instability at ion cyclotron harmonics is the nonmonotonic (loss cone or ring like) dependence of the distribution function $f$ on the perpendicular velocity $v_{\perp}$, that is, $\partial f / \partial v_{\perp}>0$. Korablev and Rudakov [1968] were the first who put forward the idea of generation of waves near the ion gyrofrequencies due to this effect. Such an instability (so called the magnetoacoustic cyclotron instability or the thermonuclear instability) was later recognized to be important in laboratory plasma because it can be driven by fast $\alpha$ particles arising as the result of thermonuclear reactions in tokamaks [Mikhailovskii, 1975]. The same mechanism for the generation of magnetosonic waves was used by Guglielmi et al. [1975] and Perraut et al. [1982] for the interpretation of magnetospheric ULF emissions. Kaladze et al. [1976] modified this approach accounting for the effects of field-aligned inhomogeneity of the geomagnetic field. They pointed out that this instability may be transformed into a kinetic (resonant) instability with a much smaller growth rate.

In a recent paper, Liu et al. [1994a] observed ULF emissions deep in the equatorial plasmasphere $(L=$ 
$1.5-2.5$ ) during great magnetic storms. In contrast to previous studies the intensity of the emission was closely related to the oxygen-ion gyrofrequency and its low harmonics $(n \leq 10)$, and the phase velocity of the wave was sometimes much smaller than Alfvén velocity $c_{A}=B_{0} /\left(\mu_{0} \rho\right)^{1 / 2}\left(B_{0}\right.$ is the ambient magnetic field and $\rho$ is the plasma density). These properties differ from the theoretical approach elaborated in the above mentioned papers.

In this paper we will present an analytical model which may provide a better understanding of the generation mechanism of ion cyclotron harmonic waves during magnetic storms. In contrast to the previous approach we shall take into consideration the effects of multi-ion composition of magnetospheric plasma in the deep plasmasphere. In cold plasma approximation, similar effects were recently taken into account by $L e$ Quéau et al. [1993] in connection with ion "conics" observed by Rauch et al. [1993] on board the Aureol 3 satellite.

\section{ULF Waves in Cold Multi-ion Plasma}

Let us consider a low-frequency wave propagating in an inhomogeneous multicomponent cold plasma immersed in the external magnetic field $B_{0}$. We choose a Cartesian system of coordinates with $z$ axis along the ambient magnetic field, $x$ axis is directed along the waveguide axis, and $y$ axis completes the triad. Small oscillations of the electromagnetic field are described by the following wave equation obtained from Faraday's and Ampere's laws [see Stix, 1962]:

$$
\nabla \nabla \cdot \mathbf{E}-\nabla^{2} \mathbf{E}+\mu_{0} \frac{\partial^{2} \mathbf{D}}{\partial t^{2}}=\mathbf{0}
$$

where $\mathbf{E}$ is the wave electric field, $\mathbf{D}=\varepsilon_{0} \mathbf{E}+(i / \omega)$ $\sum e_{j} N_{j} \mathbf{v}_{j}$ is the electric displacement, $\omega$ is the wave frequency, $e_{j}, N_{j}$, and $v_{j}$ are the electric charge, number density, and velocity of each species, respectively, and $\varepsilon_{0}$ and $\mu_{0}$ stand for the permittivity and permeability of free space. Summation is carried out over particle species.

In accordance with the results of Liu et al. [1994a, b] we restrict our study to the frequency range $\Omega_{\mathrm{H}^{+}} \gg$ $\omega \geq \Omega_{\mathrm{O}^{+}}$, where $\Omega_{\mathrm{H}^{+}}$and $\Omega_{\mathrm{O}^{+}}$are the proton and oxygen gyrofrequency, respectively. In this limit we can neglect the electron inertia perpendicular to the external magnetic field direction. Thus the electrons only undergo the $\mathbf{E} \times \mathbf{B}_{0}$ drift across the ambient magnetic field and fast motion along the magnetic field at a velocity $v_{e z}=-\left(i e / \omega m_{e}\right) E_{z}$, where $m_{e}$ is the electron mass and $e$ is the magnitude of electronic charge. On the other hand, because of the high mobility of electrons, we can neglect the motion of protons and oxygen ions along the magnetic field. The velocities of protons and oxygen ions perpendicular to the magnetic field in this approximation are equal to

$$
\mathbf{v}_{\perp \mathbf{H}^{+}}=\frac{\mathbf{E} \times \hat{\mathbf{z}}}{B_{0}}-i \frac{\omega}{\Omega_{\mathrm{H}^{+}}} \frac{\mathbf{E}}{B_{0}}
$$

$$
\mathbf{v}_{\perp \mathrm{O}^{+}}=\frac{i \Omega_{\mathrm{O}^{+}}}{\omega B_{0}} \frac{\left[\mathbf{E}+i \frac{\Omega_{\mathrm{O}^{+}}}{\omega}(\mathbf{E} \times \hat{\mathbf{z}})\right]}{1-\frac{\Omega_{\mathrm{O}^{+}}^{2}}{\omega^{2}}}
$$

where $\hat{\mathbf{z}}=\mathbf{B}_{0} / B_{0}$ is the unit vector along the geomagnetic field.

Similarly to Le Quéau et al. [1993], substituting (2) and (3) into (1), thus restricting ourselves in the following to the case of two dominant ions (protons and oxygens), we obtain

$$
\begin{gathered}
\frac{d^{2} E_{y}}{d \zeta^{2}}+\left(\varepsilon_{\perp}-n_{\perp}^{2}\right) E_{y}+i g E_{x}=0 \\
\frac{d}{d \zeta}\left[\frac{1}{1-\frac{n_{\perp}^{2}}{\varepsilon_{\|}}} \frac{d E_{x}}{d \zeta}\right]+\varepsilon_{\perp} E_{x}-i g E_{y}=0 \\
E_{z}=\frac{i n_{\perp}}{\varepsilon_{\|}\left(1-\frac{n_{\perp}^{2}}{\varepsilon_{\|}}\right)} \frac{d}{d \zeta} E_{x}
\end{gathered}
$$

Here $\zeta=\omega z / c$ is the field-aligned coordinate normalized by the vacuum wave length, $c$ stands for the velocity of light, $n_{\perp}=k_{\perp} c / \omega$ is the refractive index perpendicular to $\mathbf{B}_{0}$, and the dielectric constants $\varepsilon_{\perp}, g$, and $\varepsilon_{\|}$are defined by the relations

$$
\begin{gathered}
\varepsilon_{\perp}=\frac{c^{2}}{c_{A H}^{2}} \frac{\omega^{2}-\omega_{b i}^{2}}{\omega^{2}-\Omega_{\mathrm{O}^{+}}^{2}} \\
g=-\frac{c^{2}}{c_{A H}^{2}} \frac{\omega\left(\omega_{c o}-\Omega_{\mathrm{O}^{+}}\right)}{\omega^{2}-\Omega_{\mathrm{O}^{+}}^{2}} \\
\varepsilon_{\|}=1-\frac{\omega_{p e}^{2}}{\omega^{2}}
\end{gathered}
$$

Here $c_{A H}=B_{0} /\left(\mu_{0} N_{\mathrm{H}^{+}} m_{\mathrm{H}^{+}}\right)^{1 / 2}$ is the Alfvén velocity defined by the proton mass density, $\omega_{b i}=\Omega_{\mathrm{O}}+(1+\eta)^{1 / 2}$ is the bi-ion frequency $\left(n_{\perp}=\infty\right), \omega_{c o}=\Omega_{\mathrm{O}^{+}}(1+$ $\eta)$ is the cut-off frequency $\left(n_{\perp}=0\right), \eta=\left(N_{\mathrm{O}^{+}} m_{\mathrm{O}^{+}}\right.$ $\left./ N_{\mathrm{H}^{+}} m_{\mathrm{H}^{+}}\right)$is the ratio of the oxygen and proton mass densities, and $\omega_{p e}=\left(N_{e} e^{2} / \varepsilon_{0} m_{e}\right)^{1 / 2}$ is the Langmuir frequency. The quasi-neutrality condition yields to the equation $N_{e}=N_{\mathrm{H}^{+}}+N_{\mathrm{O}+}$. The appearance of the bi-ion frequency is connected with the existence of polarization proton drift (the second term on right-hand side of (2)) in a multicomponent plasma.

The components of the perturbed magnetic field can be easily found from Faraday's law to be

$$
\begin{gathered}
B_{z}=\frac{n_{\perp}}{c} E_{y} \\
B_{x}=\frac{i}{c} \frac{d}{d \zeta} E_{y} \\
B_{y}=-\frac{i}{c\left(1-\frac{n_{\perp}^{2}}{\varepsilon_{\|}}\right)} \frac{d}{d \zeta} E_{x}
\end{gathered}
$$

If $d / d \zeta=0$ (flute perturbations), then the general dispersion equations are separated into two parts: the ordinary wave 


$$
\omega^{2}=k_{\perp}^{2} c^{2}+\omega_{p e}^{2}
$$

and the extraordinary wave

$$
n_{\perp}^{2}=\frac{c^{2}}{c_{A H}^{2}} \frac{\omega^{2}-\omega_{c o}^{2}}{\omega^{2}-\omega_{b i}^{2}}
$$

The ordinary wave is termed so because the magnetic field does not influence the propagation of this wave in a cold plasma; that is, it propagates as an "ordinary" wave in a plasma without external magnetic field.

Figure 1 displays the dependence of transverse refractive index versus frequency for the extraordinary wave, and Figure 2 shows its dispersion. We see that in a multicomponent plasma the dispersion equation for this wave separates into two modes: a sub-Alfvénic wave which has a resonance at the bi-ion frequency $\omega_{b i}$ and a super-Alfvénic wave with a cut-off frequency at $\omega_{c o}$. The latter mode is separated from the first by an evanescent band. For small $k_{\perp}$ the phase velocity of the subAlfvénic mode equals the ordinary Alfvén velocity and then exhibits a strong dispersion with increasing wave number. In this case it displays a significant decrease in phase velocity. The upper branch also undergoes a decrease in phase velocity, but it is always higher than Alfvén velocity.

For strictly perpendicular propagation the perturbation of the magnetic field is only localized in the $B_{z}$ component which is probably not the general case in the observational data [e.g., Liu et al., 1994a]. Nevertheless, such simplification of the model allows us to understand the general features of the generation mechanism. The peculiarities of the oblique propagation are considered in section 4 .

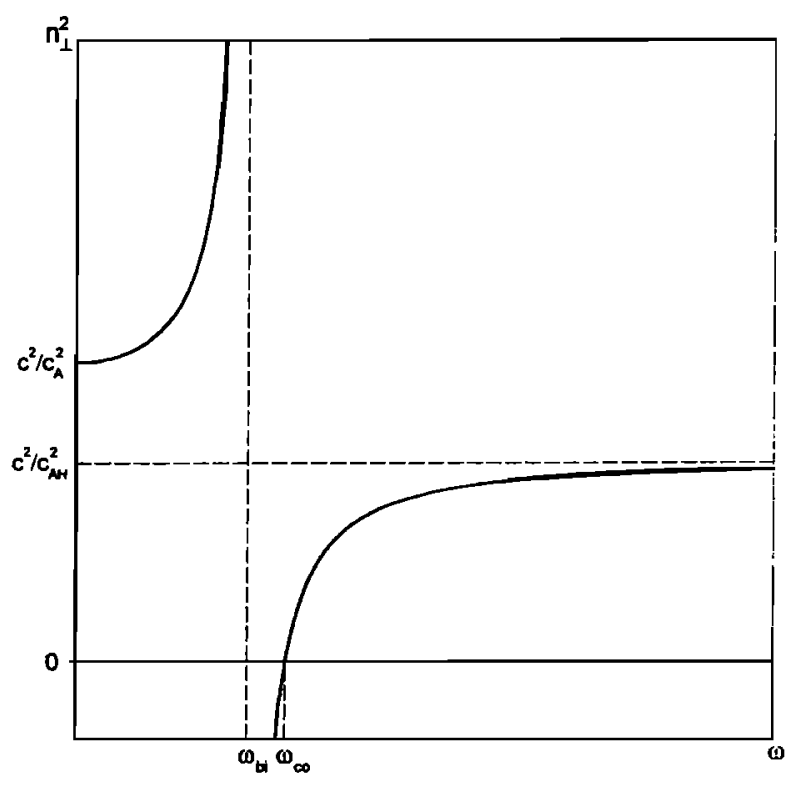

Figure 1. The dependence of the perpendicular refractive index $n_{\perp}$ versus frequency $\omega$ in case of the extraordinary wave.

\section{Instability Growth Rate}

Let us now consider the generation of extraordinary waves in a multicomponent plasma containing a small mixture of hot particles. The dispersion equation of these waves at $k_{z}=0$ may be written in the form [see Guglielmi and Pokhotelov, 1996]

$$
n_{\perp}^{2}=\frac{\varepsilon_{x x} \varepsilon_{y y}-\varepsilon_{x y} \varepsilon_{y x}}{\varepsilon_{x x}}
$$

Here the components of the dielectric tensor are expressed in the form $\varepsilon_{i j}=\varepsilon_{i j}^{c}+\varepsilon_{i j}^{h}$, where the superscripts $c$ and $h$ refer to the contribution of cold and hot plasma, respectively. On one hand, the components of the dielectric tensor from cold plasma are $\varepsilon_{x x}^{c}=\varepsilon_{y y}^{c}=\varepsilon_{\perp}$ and $\varepsilon_{x y}^{c}=-\varepsilon_{y x}^{c}=i g$. On the other hand, the components of the dielectric tensor from hot plasma may be written in the form [Guglielmi and Pokhotelov, 1996]

$$
\begin{gathered}
\varepsilon_{x x}^{h}=1+\sum_{j} \frac{\omega_{p j}^{2}}{k_{\perp}^{2} \omega} \sum_{n} \frac{n^{2} \Omega_{j}^{2}}{\omega-n \Omega_{j}} \int_{0}^{\infty} d v_{\perp} \frac{\partial f}{\partial v_{\perp}} J_{n}^{2}\left(\xi_{j}\right) \\
\varepsilon_{y y}^{h}=1+\sum_{\jmath} \frac{\omega_{p j}^{2}}{\omega} \sum_{n} \frac{1}{\omega-n \Omega_{j}} \int_{0}^{\infty} d v_{\perp} \frac{\partial f}{\partial v_{\perp}} v_{\perp}^{2} J_{n}^{\prime 2}\left(\xi_{j}\right) \\
\varepsilon_{x y}^{h}=-\varepsilon_{y x}^{h}=i \sum_{j} \frac{\omega_{p j}^{2}}{k_{\perp} \omega} \sum_{n} \frac{n \Omega_{j}}{\omega-n \Omega_{j}} \\
\times \int_{0}^{\infty} d v_{\perp} \frac{\partial f}{\partial v_{\perp}} v_{\perp} J_{n}\left(\xi_{j}\right) J_{n}^{\prime}\left(\xi_{j}\right)
\end{gathered}
$$

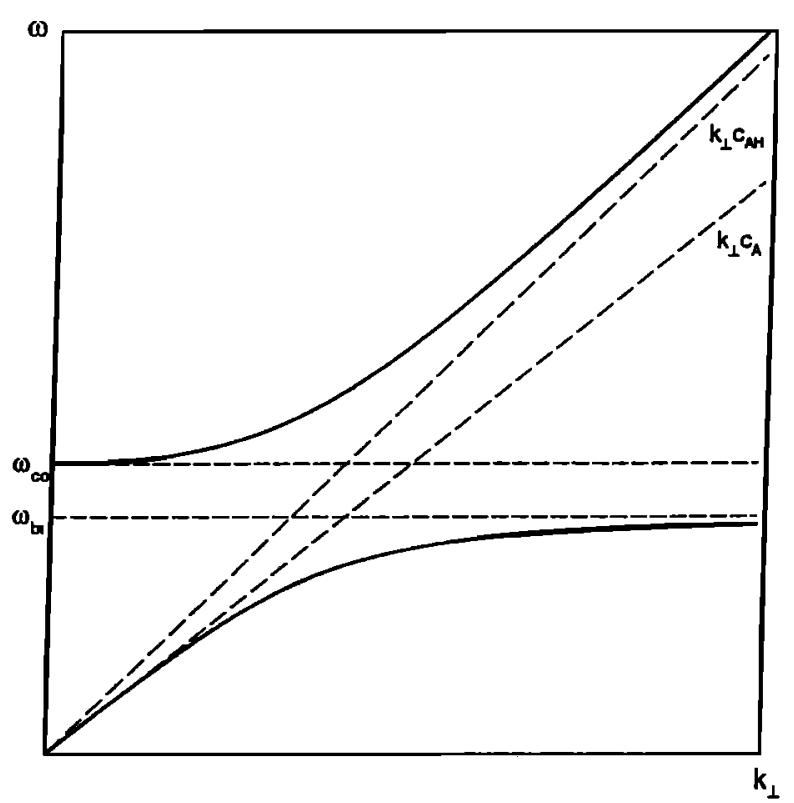

Figure 2. The dispersion of sub-Alfvénic and super-Alfvénic extraordinary waves in a multicomponent magnetospheric plasma. Here $c_{A H}=$ $B_{0} /\left(\mu_{0} N_{\mathrm{H}^{+}} m_{\mathrm{H}^{+}}\right)^{1 / 2}$ is the Alfvén velocity defined by proton mass density. 
where $\omega_{p j}=\left(n_{j} e^{2} / \varepsilon_{0} m_{j}\right)^{1 / 2}$ is the plasma frequency of $j$ species, $\Omega_{j}$ is its gyrofrequency, $n_{j}$ is the mass density of hot particles, $J_{n}$ denotes Bessel functions, $\xi_{j}=k_{\perp} v_{\perp} / \Omega_{j}$, and $\int_{0}^{\infty} f v_{\perp} d v_{\perp}=1$. Summation is carried out over all particle species.

Taking into account that the number density of hot particles is small relative to the number density of cold plasma, we may assume that $\varepsilon_{i j}^{h} / \varepsilon_{i j}^{c} \ll 1$. Thus we have the dispersion equations

$$
\begin{gathered}
D(\omega)=\varepsilon_{y y}^{h}+\frac{g^{2}}{\varepsilon_{\perp}^{2}} \varepsilon_{x x}^{h}+\frac{2 i g}{\varepsilon_{\perp}} \varepsilon_{x y}^{h} \\
D(\omega)=n_{\perp}^{2}-\frac{c^{2}}{c_{A H}^{2}} \frac{\omega^{2}-\omega_{c o}^{2}}{\omega^{2}-\omega_{b i}^{2}}
\end{gathered}
$$

Opposite to the case considered by Guglielmi et al. [1975] when the main contribution of hot particles only comes from the $\varepsilon_{x x}^{h}$ component, it is now necessary to take into account all components of the dielectric tensor. After some straightforward calculations we obtain

$$
\begin{aligned}
D(\omega)= & \frac{n_{\mathrm{O}^{+}}}{N_{\mathrm{O}^{+}}} \frac{c^{2}\left(\omega_{c o}-\Omega_{\mathrm{O}^{+}}\right)\left(\omega^{2}-\omega_{b i}^{2}\right)}{\omega\left(\omega-n \Omega_{\mathrm{O}^{+}}\right)\left(\omega^{2}-\omega_{c o}^{2}\right)} \\
& \times \int_{0}^{\infty}\left(\frac{\xi J_{n}^{\prime}}{n}-\frac{g}{\varepsilon_{\perp}} J_{n}\right)^{2} \frac{\partial f}{\partial v_{\perp}} d v_{\perp}
\end{aligned}
$$

where $n_{\mathrm{O}^{+}}$stands for the hot oxygen ion number density.

Near the oxygen harmonics $\omega \approx n \Omega_{\mathrm{O}^{+}}$, we make use of the expansion

$$
D(\omega) \approx D(\omega)_{\omega=n \Omega_{\mathrm{O}^{+}}}+\left(\omega-n \Omega_{\mathrm{O}^{+}}\right)\left(\frac{\partial D}{\partial \omega}\right)_{\omega=n \Omega_{\mathrm{O}^{+}}}
$$

(Generally speaking, in the near vicinity of the harmonics of the gyrofrequency it is necessary to take the thermal effects of the background plasma into account. However, the corresponding frequency shift caused by the thermal effects is much narrower than the width of the resonance peak [e.g., Guglielmi et al., 1975]. Therefore we can use dispersion equation (20) and definition (22) for the growth rate everywhere outside a very narrow band in the vicinity of gyrofrequency harmonics.) According to the cold plasma approximation, $D(\omega)_{\omega=n \Omega_{\mathrm{O}^{+}}}=0$. Now supposing $\omega=n \Omega_{\mathrm{O}^{+}}+i \gamma_{L}$, we finally obtain the expression for the linear growth rate $\gamma_{L}$

$$
\begin{aligned}
\gamma_{L}^{2}=- & \frac{n_{\mathrm{O}^{+}}}{N_{\mathrm{O}^{+}}} \frac{c^{2} \Omega_{\mathrm{O}^{+}}\left(\omega_{c o}-\Omega_{\mathrm{O}^{+}}\right)\left(n^{2} \Omega_{\mathrm{O}^{+}}^{2}-\omega_{b i}^{2}\right)}{\left(n^{2} \Omega_{\mathrm{O}^{+}}^{2}-\omega_{c o}^{2}\right)} \\
& \times \frac{\int_{0}^{\infty}\left(\frac{\xi J_{n}^{\prime}}{n}-\frac{g}{\varepsilon_{\perp}} J_{n}\right)^{2} \frac{\partial f}{\partial v_{\perp}} d v_{\perp}}{(\omega \partial D / \partial \omega)_{\omega=n \Omega_{\mathrm{O}^{+}}}}
\end{aligned}
$$

where $(\omega \partial D / \partial \omega)_{\omega=n \Omega_{\mathrm{O}^{+}}}=-2 \frac{c^{2}}{c_{A H}^{2}}\left[1+\frac{\omega_{b i}^{2}\left(\omega_{c o}^{2}-\omega_{b i}^{2}\right)}{\left(n^{2} \Omega_{\mathrm{O}^{+}}^{2}-\omega_{b i}^{2}\right)^{2}}\right]<0$

It is evident from (22) that the growth rate becomes positive only in the case when the distribution function of hot particles has a positive slope, that is, $\partial f / \partial v_{\perp}>$ 0 . This may be realized in case of loss cone or ring like distribution of hot oxygen ions. We find the following approximate estimate for the growth rate

$$
\gamma_{L} \sim\left(\frac{n_{\mathrm{O}^{+}}}{N_{\mathrm{H}^{+}}}\right)^{1 / 2} \frac{c_{A H}}{v_{T}}\left(\frac{m_{\mathrm{O}^{+}}}{m_{\mathrm{H}^{+}}}\right)^{1 / 2} \Omega_{\mathrm{O}^{+}}
$$

where $v_{T}$ is the thermal velocity of hot oxygen ions.

In the general case, the components $\varepsilon_{2 j}^{h}$ should also take into account the effect of field-aligned inhomogeneity of the magnetic field [e.g., Kaladze et al., 1976]. The importance of this effect may be evaluated using the following simple consideration. Near the equatorial region the geomagnetic field may be expanded according to $B_{0}=B_{e q}\left[1+(9 / 2)\left(z / L R_{E}\right)^{2}\right]$, where $L$ is the Mclllwain parameter, $R_{E}$ is the Earth's radius, and $B_{e q}$ is the equatorial value of the geomagnetic field. The variation of the gyrofrequency during the particle motion then equals

$$
\frac{\Delta \Omega_{\mathrm{O}^{+}}}{\Omega_{\mathrm{O}^{+}}} \approx \frac{9}{2} \frac{v_{T}^{2}}{\left(\gamma_{L} L R_{E}\right)^{2}}
$$

Here it was estimated that $z \approx v_{T} / \gamma_{L}$. The fieldaligned inhomogeneity is negligible if $\Delta \Omega_{\mathrm{O}^{+}} \ll \Omega_{\mathrm{O}^{+}}$ or

$$
\frac{n_{\mathrm{O}^{+}}}{N_{\mathrm{H}^{+}}} \gg \epsilon=\frac{9}{2} \frac{r_{\mathrm{O}^{+}}^{2}}{\left(L R_{E}\right)^{2}} \frac{v_{T}^{2}}{c_{A H}^{2}} \frac{m_{\mathrm{H}^{+}}}{m_{\mathrm{O}^{+}}}
$$

where $r_{\mathrm{O}^{+}}=v_{T} / \Omega_{\mathrm{O}^{+}}$is the hot oxygen gyroradius. It follows from (25) that the inhomogeneity of the magnetic field may be neglected when the hot oxygen number density is sufficiently high to fulfill the inequality (25).

In the opposite case the effect of magnetic field inhomogeneity has to be taken into account. Accordingly, the gyrofrequency averaged along the field line becomes dependent on the energy $\varepsilon=v^{2} / 2$ and the magnetic moment $\mu=v_{\perp}^{2} / 2 B_{0}$ (for details see, for example, Kaladze et al. $[1976])$, that is,

$$
\bar{\Omega}_{\mathrm{O}^{+}}=\frac{\oint \Omega_{\mathrm{O}^{+}}(\varepsilon, \mu) \frac{d s}{\left|v_{\|}\right|}}{\oint \frac{d s}{\left|v_{\|}\right|}}
$$

where $v_{\|}=\left[2\left(\varepsilon-\mu B_{0}\right)\right]^{1 / 2}$ is the field-aligned particle velocity. This will result in the appearance of resonant wave-particle interaction $\left(\omega \simeq n \bar{\Omega} \gg \omega_{b}\right.$, where $\omega_{b}$ is the bounce frequency) which will lead to a weak instability with a growth rate $\gamma_{L} \propto n_{\mathrm{O}^{+}}$instead of $\gamma_{L} \propto n_{\mathrm{O}^{+}}^{1 / 2}$ as in (23). (In this case the denominator $1 /\left(\omega-n \bar{\Omega}_{\mathrm{O}^{+}}\right)$in the formular similar to (20) must be understood in Landau sense: $\lim _{\nu \rightarrow 0}\left[1 /\left(\omega+i \nu-n \bar{\Omega}_{\mathrm{O}^{+}}\right)\right]=$ $P\left[1 /\left(\omega-n \bar{\Omega}_{\mathrm{O}^{+}}\right)\right]-i \pi \delta\left(\omega-n \bar{\Omega}_{\mathrm{O}^{+}}\right)$, where $P$ denotes that the Cauchy principal value of the integral is to be 
taken at the singular point $\omega=n \bar{\Omega}_{\mathrm{O}^{+}}$.) Thus the whole scenario of the instability is as follows. At low oxygen densities when the inequality (25) fails, the oscillations grow with $\gamma_{L} \propto n_{\mathrm{O}^{+}}$. However, this instability may be easily stabilized by quasi-linear effects, for example, because of the formation of a plateau in the distribution function. When the density of hot oxygen ions exceeds a certain critical value, the waves undergo a strong amplification with the growth rate given in (23).

\section{Discussion}

Now let us apply our theory in order to interpret the ULF wave observations with discrete spectra reported by Liu et al. [1994a]. The A kebone satellite was launched in February 1989 into an elliptical orbit with an apogee of $10,500 \mathrm{~km}$, perigee of $274 \mathrm{~km}$, and inclination of $75.0^{\circ}$. Because of its low altitude, this satellite was able to monitor the region of the magnetosphere where a large amount of cold $\mathrm{H}^{+}$ions coexists with a sufficient mixture of cold $\mathrm{O}^{+}$ions of ionospheric origin. The data presented by Liu et al. [1994a] show the generation of ULF emissions with discrete spectra appearing in the deep plasmasphere at $L=1.5-2.5$ during great magnetic storms. Figure 1 of Liu et al. [1994a] shows multiband emissions which consist of harmonically spaced spectral bands near oxygen gyrofrequency harmonics. The number of harmonics $n$ was less than 10. The higher harmonics were subject to strong nonlinear interactions. It should be mentioned that Russell et al. [1970], who measured ELF emissions near proton gyrofrequency harmonics, observed high $n$ numbers.

For numerical estimates we choose the following parameters: $v_{T} \approx 10^{6} \mathrm{~ms}^{-1}, \Omega_{\mathrm{O}^{+}} \approx 25 \mathrm{~s}^{-1}, L=2$, $N_{\mathrm{H}^{+}}=2 \times 10^{-2} \mathrm{~m}^{-3}$ and $T_{\mathrm{H}^{+}}=2 \times 10^{3} \mathrm{~K}$. Substituting these values into (25) we obtain $\epsilon \approx 10^{-5}$. A reasonable value of the parameter $n_{\mathrm{O}^{+}} / N_{\mathrm{H}^{+}}$during high geomagnetic activity, for example, during magnetic storms, is of the order of $10^{-3}-10^{-4}$. Thus the inequality (25) is fulfilled, and the effects of magnetic field inhomogeneity may be neglected. On the other hand, during quiet times it is necessary to take the magnetic field inhomogeneity into account. The estimate for the growth rate of the hydrodynamic instability thus gives $\gamma_{L} \approx 1 \mathrm{~s}^{-1}$.

In the upper branch the three-wave interactions are forbidden: $\omega\left(\mathbf{k}_{1}\right)+\omega\left(\mathbf{k}_{2}\right) \neq \omega\left(\mathbf{k}_{\mathbf{1}}+\mathbf{k}_{2}\right)$ for any $\mathbf{k}_{1}$ and $\mathbf{k}_{2}$. It means that a weakly nonlinear dynamics of these oscillations is defined only by induced scattering with thermal plasma. Thus we should expect a wave energy accumulation of super-Alfvénic oscillations near the lower harmonics as it was demonstrated by Liu et al. [1994a]. The rate of the induced scattering may be estimated according to the dimensional considerations as $\gamma_{N L} \approx\left(k_{z} / k_{\perp}\right)^{2}\left[W /\left(N_{\mathrm{H}^{+}} T_{\mathrm{H}^{+}}\right)\right] \Omega_{\mathrm{O}^{+}}$, where $W$ is the wave energy density. According to Liu et al. [1994a], $\left(k_{z} / k_{\perp}\right)^{2} \approx 0.1$ and $B_{z}=0.3 \mathrm{nT}$. Using this value, we come to the conclusion that $\gamma_{N L}{ }^{-1} \gg \gamma_{L}{ }^{-1}$; that is, the rate of wave energy transfer into the region of small frequencies is low relative to the linear growth of the waves.
In order to discuss the polarization characteristics of the generated waves, let us introduce the components $B_{R}, B_{L}$, and $B_{\|}$, where $B_{R}=B_{x}-i B_{y}$ and $B_{L}=B_{x}+$ $i B_{y}$ are the components of right-handed and left-handed polarization in the plane perpendicular to the magnetic field line and $B_{\|} \equiv B_{z}$. For oblique propagation we obtain with the help of (10)-(12)

$$
\begin{gathered}
B_{R}=-i\left(\frac{k_{z}}{\omega}\right)\left[1+\frac{\omega^{2}-\omega_{b i}^{2}}{\omega\left(\omega_{c o}-\Omega_{\mathrm{O}^{+}}\right)}\right] E_{x} \\
B_{L}=i\left(\frac{k_{z}}{\omega}\right)\left[1-\frac{\omega^{2}-\omega_{b i}^{2}}{\omega\left(\omega_{c o}-\Omega_{\mathrm{O}^{+}}\right)}\right] E_{x} \\
B_{\|}=i\left(\frac{k_{\perp}}{\omega}\right)\left(\frac{\omega^{2}-\omega_{b i}^{2}}{\omega\left(\omega_{c o}-\Omega_{\mathrm{O}^{+}}\right)}\right) E_{x}
\end{gathered}
$$

where (4) and (5) were Fourier-transformed along the $z$ axis with the parallel wave number $k_{z} \ll k_{\perp}$. It follows from (27) and (28) that $\left|B_{R}\right|<\left|B_{L}\right|$ for sub-Alfvénic waves and $\left|B_{R}\right|>\left|B_{L}\right|$ for super-Alfvénic waves. This effect has a purely multicomponent origin and was observed experimentally by Liu et al. [1994a]. It should be mentioned that wave observations in a purely hydrogen plasma always found that $\left|B_{R}\right|>\left|B_{L}\right|[$ Perraut et al., 1982].

Finally, let us discuss the phase velocities of the generated waves. Liu et al. [1994a] also estimated the spectral densities $\delta E / \delta B$ of the Fourier components for some events and obtained phase velocities of several tens of kilometers per second to about $100 \mathrm{~km} \mathrm{~s}^{-1}$, which, from their point of view, is much less than the Alfven velocity in the vicinity of the Akebono orbit. According to our model the loss cone or ring like distribution of hot oxygen ions may generate two modes: super-Alfvénic and sub-Alfvénic waves. The phase velocity of the first mode is always larger than the Alfvén velocity. On the other hand, the other (sub-Alfvénic) branch undergoes a strong dispersion near the bi-ion frequency, and these oscillations may have phase velocities much smaller than the Alfvén velocity. It may be that Liu et al. [1994a] have only selected cases corresponding to sub-Alfvénic waves. For the verification of this concept it is necessary to perform a detailed ULF data analysis of Akebono satellite observations.

Now let us consider the necessary conditions for the instability. It follows from (22) that the sign of the growth rate depends on the sign of the integral $I$

$$
I=\int_{0}^{\infty}\left[\xi J_{n}^{\prime} / n-\left(g / \varepsilon_{\perp}\right) J_{n}\right]^{2} \frac{\partial f}{\partial v_{\perp}} d v_{\perp}
$$

Integrating (30) by parts, we obtain

$$
I=-\int_{0}^{\infty} f \frac{\partial\left[\xi J_{n}^{\prime} / n-\left(g / \varepsilon_{\perp}\right) J_{n}\right]^{2}}{\partial v_{\perp}} d v_{\perp}
$$

For the occurrence of the instability it is necessary that $I>0$. Such a situation may appear in the case of "loss cone" or "ring like" distribution of the hot oxygen 
ions. Because we do not know the actual form of the distribution function, we may choose the simplest case of ring like distribution

$$
f=\left(2 \pi v_{\perp 0}\right)^{-1} \delta\left(v_{\perp}-v_{\perp 0}\right)
$$

where $v_{\perp 0}$ is the oxygen ion "ring" velocity in the perpendicular direction. According to Geos 1 observations [Perraut et al., 1982] the appearance of proton harmonics coincided with the occurrence of so-called proton rings with the distribution function similar to (32). It should be mentioned that these ring like distribution functions for protons were not systematically observed when magnetosonic waves were detected [Perraut et al., 1982]. Occasionally, loss cone distributions were also observed which can also provide the free energy for extraordinary waves.

Substituting (32) into (31), we have

$$
I=-\left.\frac{\partial\left[\xi J_{n}^{\prime} / n-\left(g / \varepsilon_{\perp}\right) J_{n}\right]^{2}}{2 \pi v_{\perp} \partial v_{\perp}}\right|_{v_{\perp}=v_{\perp} 0}
$$

$I$ is negative for small arguments $\xi_{0}=k_{\perp} v_{\perp 0} / \Omega_{\mathrm{O}^{+}} \ll 1$ and becomes positive at sufficiently high ring velocity $v_{\perp 0}$. In order to obtain an explicit expression for a marginal instability, let us consider the limiting case of a narrow stop band when $\eta \ll 1$. In this case the expression (33) reduces to

$$
I=\frac{n}{\pi v_{p h} v_{\perp 0}}\left(\frac{v_{\perp 0}}{v_{p h}}\right)^{2} J_{n} J_{n}^{\prime}\left(1-\left(\frac{v_{p h}}{v_{\perp 0}}\right)^{2}\right)
$$

where $v_{p h}$ is the phase velocity of oscillations. The instability occurs when $v_{\perp 0}>v_{p h}$, that is, when the ring velocity becomes larger than the wave phase velocity. This is similar to the Cherenkov radiation condition. When $v_{\perp 0} / v_{p h}$ is large, the envelope of the growth rate is oscillating. These oscillations reflect interferences between Bessel functions. We consider them as unphysical because they would not appear with smooth distribution functions. It should be mentioned that the reversal of the sign of $J_{n} J_{n}^{\prime}$ occurs at larger values of $v_{\perp 0}$.

\section{Conclusions}

Let us now summarize the main results of our theory. The recent experimental study of ULF emissions detected on board the Akebone satellite gives a useful background for investigations of magnetospheric plasma stability at low altitudes. These emissions are strongly affected by ionospheric plasma, and one should expect to observe a significant role of $\mathrm{O}^{+}$ions in the wave dynamics. The initial observations of these emissions made by Kokubun et al. [1991] during relatively quiet magnetic activity demonstrated their close correlation with $\mathrm{He}^{+}$gyrofrequency and its harmonics. According to our model these oscillations may result in the generation of loss cone or ring like resonant instability with $\gamma_{L} \propto n_{\mathrm{He}^{+}}$, leading to the excitation of ULF emissions near the helium ion cyclotron frequency and its harmonics. During great magnetic storms, when a sufficiently high mixture of ionospheric $\mathrm{O}^{+}$ions is observed, a switch to a stronger instability with $\gamma \propto\left(n_{\mathrm{O}^{+}}\right)^{1 / 2}$ is possible. The latter instability may generate both sub-Alfvénic and super-Alfvénic waves near oxygen gyrofrequency harmonics. These waves undergo complex nonlinear interactions which can be studied by means of detailed data analysis.

Acknowledgments. This research was partially supported by the Commission of the European Union (Brussels) through research grant INTAS-94-2811. The authors also acknowledge the support of the Russian Fund for Fundamental Research through grant 96-05-64445 and the Academy of Finland. Two of us (O.A.P. and V.A.G.) wish to thank the French Ministère de la Recherche et de la Technologie for hospitality at LPCE. The authors are grateful to V. Krasnosel'skikh for helpful discussions.

The Editor thanks Susumu Kokubun and Dominique LeQuéau for their assistance in evaluating this paper.

\section{References}

Guglielmi, A. V., and O. A. Pokhotelov, Geoelectromagnetic Waves, IOP, Bristol, Pa., 1996.

Guglielmi, A. V., B. I. Klaine, and A. S. Potapov, Excitation of magnetosonic waves with discrete spectrum in the equatorial vicinity of the plasmapause, Planet. Space Sci., 23, 279, 1975.

Gurnett, D. A., Plasma wave interaction with energetic ions near the magnetic equator, $J$. Geophys. Res., 81, 2765, 1976.

Gurnett, D. A., and U. S. Inan, Plasma waves observations with the Dynamics Explorer 1 spacecraft, Rev. Geophys., 26, 285, 1988.

Kaladze T. D., A. B. Mikhailovskii, A. S. Potapov, and O. A. Pokhotelov, Role of longitudinal magnetic field inhomogeneity in cyclotron instability of the plasmasphere, Sov. J. Plasma Phys., Engl. Transl., 2, 370, 1976.

Kintner, P. M., and D. A. Gurnett, Observations of ion cyclotron waves within the plasmasphere by Hawkeye 1, $J$. Geophys. Res., 82, 2314, 1977.

Kokubun, S., M. Takami, K. Hayashi, H. Fukunishi, I. Kimura, A. Sawada, and Y. Kasahara, Triaxial search coil measurements of ELF waves in the plasmasphere: Initial results from EXOS-D, Geophys. Res. Lett., 18, 301, 1991.

Korablev, L. V., and L. I. Rudakov, Instability of a plasma with an isotropic distribution function, Sov. Phys. JETP, Engl. Transl., 27, 439, 1968.

Korth, A., G. Kremser, S. Perraut, and A. Roux, Interaction of particles with ion cyclotron waves and magnetosonic waves: Observations from GEOS 1 and GEOS 2, Planet. Space Sci., 32, 1393, 1984.

Laakso, H., H. Juginger, A. Roux, R. Schmidt, and C. de Villedary, Magnetosonic waves above $f_{c}\left(H^{+}\right)$at geostationary orbit: GEOS 2 results, J. Geophys. Res., 95, $10609,1990$.

Le Quéau, D., A. Roux, J. L. Rauch, F. Lefeuvre, and J. M. Bosqued, Heating of protons by resonant absorption in a multicomponent plasma, 2, Theoretical model, J. Geophys. Res., 98, 13363, 1993.

Liu, H., S. Kokubun, and K. Hayashi, Equatorial electromagnetic emission with discrete spectra near harmonics of oxygen gyrofrequency during magnetic storm, Geophys. Res. Lett., 21, 225, 1994a.

Liu, H., K. Hayashi, and S. Kokubun, Gyroharmonic electromagnetic emissions induced by oxygen ion $\left(\mathrm{O}^{+}\right)$in the equatorial plasmasphere during magnetic storms, paper presented at Eighth International Symposium on Solar Terrestrial Physics, Sendai, Japan, June 5-10, 1994 b. 
McClements, K. G., Ion cyclotron harmonic waves in the equatorial magnetosphere, Adv. Space Res., 17(10), 73, 1996.

Mikhailovskii, A. B., Electromagnetic instabilities in a nonMaxwellian plasma, Reviews of Plasma Physics, vol. 6, pp. 77-159, edited by M. A. Leontovich, Consult. Bur., New York, 1975.

Olsen, R. C., S. D. Shawhan, D. L. Gallagher, J. L. Green, C. R. Chappel, and R. R. Anderson, Plasma observations at the Earth's magnetic equator, J. Geophys. Res., 92, 2385, 1987.

Perraut, S., R. Gendrin, P. Robert, A. Roux, C. de Villedary, and D. Jones, ULF waves observed with magnetic and electric sensors on GEOS 1, Space Sci. Rev., 22, 347, 1978.

Perraut, S., A. Roux, P. Robert, R. Gendrin, J. A. Sauvaud, J. M. Bosqued, G. Kremser, and A. Korth, A systematic study of ULF waves above $F_{H^{+}}$from GEOS 1 and 2 measurements and their relationship with proton ring distributions, J. Geophys. Res., 87, 6219, 1982.

Rauch, J. L., F. Lefeuvre, D. Le Quéau, A. Roux, J. M. Bosqued, and J. J. Berthelier, Heating of protons by resonant absorption in a multicomponent plasma, 1, Experimental evidence, J. Geophys. Res., 98, 13347, 1993.
Russell, C. T., R. E. Holzer, and E. Smith, OGO 3 observations of ELF noise, $J$. Geophys. Res., 75, 755, 1970.

Stix, T. H., The Theory of Plasma Waves, Mc Graw-Hill, New York, 1962.

Taylor, W. L., B. K. Parady, and L. J. Cahil Jr., Explorer 45 observations of $1-$ to $30-\mathrm{Hz}$ magnetic fields near the plasmapause during magnetic storms, J. Geophys. Res., 80, 1271, 1975.

F. Z. Feygin, V. A. Gladychev, D. O. Pokhotelov, and O. A. Pokhotelov, Institute of Physics of the Earth, 123810 Moscow, Russia (e-mail: pokh@iephys.msk.su)

K. Hayashi, Department of Earth and Planetary Physics, University of Tokyo, Tokyo 113, Japan (e-mail: hayashi@grl.s.u-tokyo.ac.jp)

J. Kangas and K. Mursula, Department of Physical Sciences, University of Oulu, P.O. Box 333, FIN90571 Oulu, Finland. (e-mail: jorma.kangas@oulu.fi; kalevi.mursula@oulu.fi)

M. Parrot, Laboratoire de Physique et Chimie de l'Environnement, CNRS, 45071 Orléans Cedex 2, France.

(Received July 16, 1996; revised September 27, 1996; accepted October 3, 1996.) 